\title{
PRIMARY STRUCTURE OF A B1 HORDEIN GENE FROM BARLEY
}

\author{
by
}

\author{
ANDERS BRANDT, ALAIN MONTEMBAULT", VERENA CAMERON-MILLS \\ and \\ SØREN K. RASMUSSEN ${ }^{21}$
}

Department of Physiology, Carlsberg Laboratory

Gamle Carlsberg Vej 10, DK-2500 Copenhagen Valby

Present address: "Laboratoire Tepral, 2, rue Gabriel Bour,

F.54250 Champigneulles, France

${ }^{2}$ Agricultural Research Department, Risø National Laboratory,

DK-4000 Roskilde, Denmark

Keywords: Endosperm, storage protein, Hordeum vulgare, gene family, heterogeneity,
molecular cloning

The 873 base pair coding region of a Hor- 2 gene of barley and the adjacent 550 base pair upstream and 413 base pair downstream regions were sequenced. The gene is devoid of introns and encodes a 271 amino acid long B1 hordein polypeptide containing a putative 19 amino acid signal peptide. The remaining part of the coding sequence can be divided into three parts. In the 53 residue amino-terminal region there are 9 glutamine-proline blocks with a preferred core sequence $\mathrm{PQQP}$, separated by one or two other residues giving a glutamine proline content of $78 \%$. The second part encodes 164 amino acids, $41 \%$ of which are glutamine + proline organised in scattered blocks. Seven cysteine residues are coded for by this portion of the gene. The last part encodes the carboxyterminal 35 amino acids none of which is glutamine.

In the 550 base pair upstream region the sequence TATAAATA is found at -71 base pairs from the initiator methionine. In the 3' non-coding region three putative polyadenylation signals, AATAAA, are present.

Comparison of the gene with 3 partial CDNA clones indicates that the charge polymorphism in the B1 polypeptide group is due to point mutations in the part of the gene corresponding to the carboxy terminal half of the polypeptide. Comparison with the sequence of a second $B$ hordein gene suggests that insertions or deletions of glutamine-proline blocks in the amino-terminal domain are a major source of size polymorphisms in the B hordein family.

Abbreviations: $b p=$ base pair; $k b p=$ kilo base pair 


\section{INTRODUCTION}

Chromosome 5 in barley contains two complex loci Hor- 1 and Hor- 2 encoding the $C$ and $B$ hordein polypeptides, respectively $(21,36)$. These are the major storage proteins of the barley grain. Each group of storage proteins comprises a number of related polypeptides which differ in molecular weight, charge and peptide map $(9,18)$. The $B$ hordein polypeptides comprise 3 size classes, as shown by SDS gel electrophoresis, but further separation by isoelectric focussing identifies a number of isoelectric variants in each size class (9). A number of B hordein cDNA clones have been characterized and the amino acid sequences derived from them have shown the polypeptides to comprise three distinct domains $(11,30)$. The amino-terminal domain 1 contains permutated blocks of 4 to 6 proline and glutamine residues separated by a single or two other amino acids. In domain 2 blocks of glutamine and proline residues are more widely spaced and the presence of cysteine + methionine is a characteristic feature. Comparison of CDNA clones indicates that variation in the domain 2 is due to amino acid substitutions as well as individual amino acid deletions. These may account for the isoelectric variants seen in each size class. The reason for the size variation within the B hordein family has yet to be established.

The gene copy number derived from Southern blot analysis of barley DNA indicates that the B hordein gene family is composed of 13 members (22). The polypeptides are synthesized 15-25 days after fertilization in the developing endosperm cell on membrane-bound polysomes of the endoplasmic reticulum as larger sized precursors and co-translationally transported across the membrane concomitant with signal peptide cleavage $(3,6,40)$. They are ultimately deposited in the vacuole where they precipitate out as protein bodies $(5,7)$. Upon extraction, the B hordein polypeptides will aggregate if a reducing agent is omitted in the extraction media, due to the high amount (3\%) of cysteine (33). Ultrastructural analysis of seeds of a mutant (hor2ca) defective in B hordein synthesis has shown that there is a failure of the storage polypeptides to package into condensed protein bodies (7). Owing to their ability to form intra- and intermolecular disulfide bridges it is likely that the B hordein polypeptides are responsible for the proper packaging of protein in the vacuole. In contrast, the $C$ hordein polypeptides have a low amount of cysteine. $\mathrm{C}$ hordein is also particularly enriched in glutamine and proline, the $\mathrm{C} 2$ hordein polypeptide containing - $70 \%$ of these residues, as compared to a $\sim 50 \%$ content in the B1 hordein (33). Knowledge of the primary structure of the storage polypeptides is essential in order to investigate the packaging process.

The expression of both gene families in the endosperm can be enhanced by the supply of nitrogen in the form of ammonium nitrate to cut developing spikes $(14,15)$. The expression of $\mathrm{C}$ hordein genes increases linearly in response to increasing nitrogen supply. Although B hordein gene expression responds linearly to low levels of increasing nitrogen supply the response reaches saturation at levels far below the $\mathrm{C}$ hordein expression maximum. One might search for conserved nucleotide sequences of 5' and 3' flanking regions of different hordein genes to identify regulatory sequences responsible for controlling gene expression with respect to tissue specificity, temporal specificity and nitrogen regulation.

As a step towards this goal a genomic clone encoding a B1 hordein polypeptide has been isolated and sequenced.

\section{MATERIALS AND METHODS}

\subsection{Chemicals}

All restriction endonucleases, T4 DNA ligase, DNA polymerase 1 (Klenow fragment), 2-deoxy and 2'3' dideoxy nucleotide triphosphates were from Boehringer, Mannheim (FRG), $\alpha-{ }^{32} \mathrm{P}-\mathrm{ATP}$ from New England Nuclear (USA).

\subsection{Construction and screening of an Eco RI barley library}

Barley DNA was isolated from immature endosperms of Hordeum vulgare c.v. Carlsberg II according to HopP et al. (19). The DNA was partially digested with Eco RI and the resulting fragments ligated to Eco RI, Hind III and Sal I digested $\lambda$ L47.1 DNA (23), in vitro packaged 
(17) and plated onto the rec $\mathrm{BC}^{-}$strain BJS183 (37). The yield of recombinant phages was estimated, by plating onto a P2 host, to be $10^{4} \cdot \mu \mathrm{g}^{-1}$ of $\lambda$ DNA. The library was screened according to BENTON and DAVIS (2) using the nick-translated pc hor2-4 (30) as a probe. Small or large scale preparation of phage DNA was according to MANIATIS et al. (26). The isolated genomic clone was mapped by Southern blot hybridization analysis.

\subsection{DNA sequencing by the dideoxy chain termination method}

The hybridizing fragments found in the isolated genomic clone were subcloned in pBR 328 (8) using HB 101 as host. Restriction fragments from these subclones were isolated by agarose gel electrophoresis and inserted into the bacteriophage $\mathrm{M} 13$ vectors (27) $\mathrm{mp} 8$ and $\mathrm{mp} 9$ and cloned by transformation of JM 101 cells. Random cloning was also performed from a restriction digest of the pBR 328 subcloned inserts. Media, strains, chemicals, gel preparation and sequencing of subclones by the dideoxy chain termination method were as described in $\mathbf{3 0}$, 32) except that the sequencing reactions were performed at $45^{\circ} \mathrm{C}$. The nucleotide sequence data was processed on a Honeywell DPS-8 using a program supplied by Dr. R. STADEN, MRC, Cambridge, England.

The universal primer for sequencing in M13 was standardly used, except when sequences longer than 300 bp were to be determined. In 4 cases specific 14 mer oligonucleotides were synthesized by phosphoramidite chemistry on an Applied Biosystem 380A DNA synthesizer using the protocol supplied by the company.

\section{RESULTS}

\subsection{Identification and restriction map of the clone $\lambda$ hor 2-4}

Partial Eco RI restriction fragments of Carlsberg II barley DNA were cloned into the vector $\lambda$ L47.1. Upon screening of 200,000 plaques with the $\mathrm{B}$ hordein cDNA probe pc hor2-4, one strongly hybridizing plaque was purified and analysed by Southern blot analysis. The clone $(\lambda$ hor2-4) contains two hybridizing Eco RI frag- ments of 3.5 and $1.2 \mathrm{kbp}$ (Figure 1B), corresponding in size to the two smallest Eco RI fragments seen in Southern blot analysis of Carlsberg II DNA using the same pc hor 2-4 probe (Figure 1A). When the clone was digested with the restriction endonuclease Hind III only one fragment of $2.9 \mathrm{kbp}$ hybridized to the probe, corresponding in size to one Hind III fragment in Carlsberg II DNA. Estimation of gene copy number for the Hor-2 locus from the Southern blot (Figure 1A) indicates a gene family of more than 10 members. The hybridizing Eco RI and Hind III restriction fragments were subcloned in pBR328 and further mapping performed with combinations of different restriction enzymes. The derived map is shown in Figure 2. Since the two Eco RI fragments and the Hind III fragment of the clone are similar in size to the fragments present in barley DNA we conclude that the cloned fragments have not been subjected to major rearrangements during the cloning procedure.

\subsection{Nucleotide sequence determination}

Restriction fragments from the 2.9 Hind III fragment generated by the enzymes Taq 1, Alu I, Rsa I, Sau 3A, Acc I and Eco RI were cloned into M13 mp8 and $\mathrm{mp} 9$ and sequenced by the dideoxy chain termination method using the universal primer. Sequencing capacity in longer fragments was extended by priming the sequencing reaction with synthetic oligonucleotides. The obtained sequences were confirmed by restriction analysis and the fine map of the 1775 bp sequence, as well as the sequencing strategy, are shown in Figure 3.

The 1775 bp sequence (Figure 4) encodes a 271 amino acid long $\mathbf{B} 1$ hordein polypeptide. The 164 carboxy-terminal amino acids deducible from the clone are identical to those predicted by partial cDNA clone, pc hor2-4 (30). The latter clone was shown to code for a B1 hordein polypeptide by alignment with six $\mathrm{B} 1$ hordein peptide sequences established by automated Edman degradation (34). The genomic clone is also identical with the cDNA nucleotide sequence with the exception of position 353, in which a $C$ is found instead of an $A$. We conclude that the cDNA was copied from the mRNA of the 
A. BRANDT et al:: Bl hordein gene
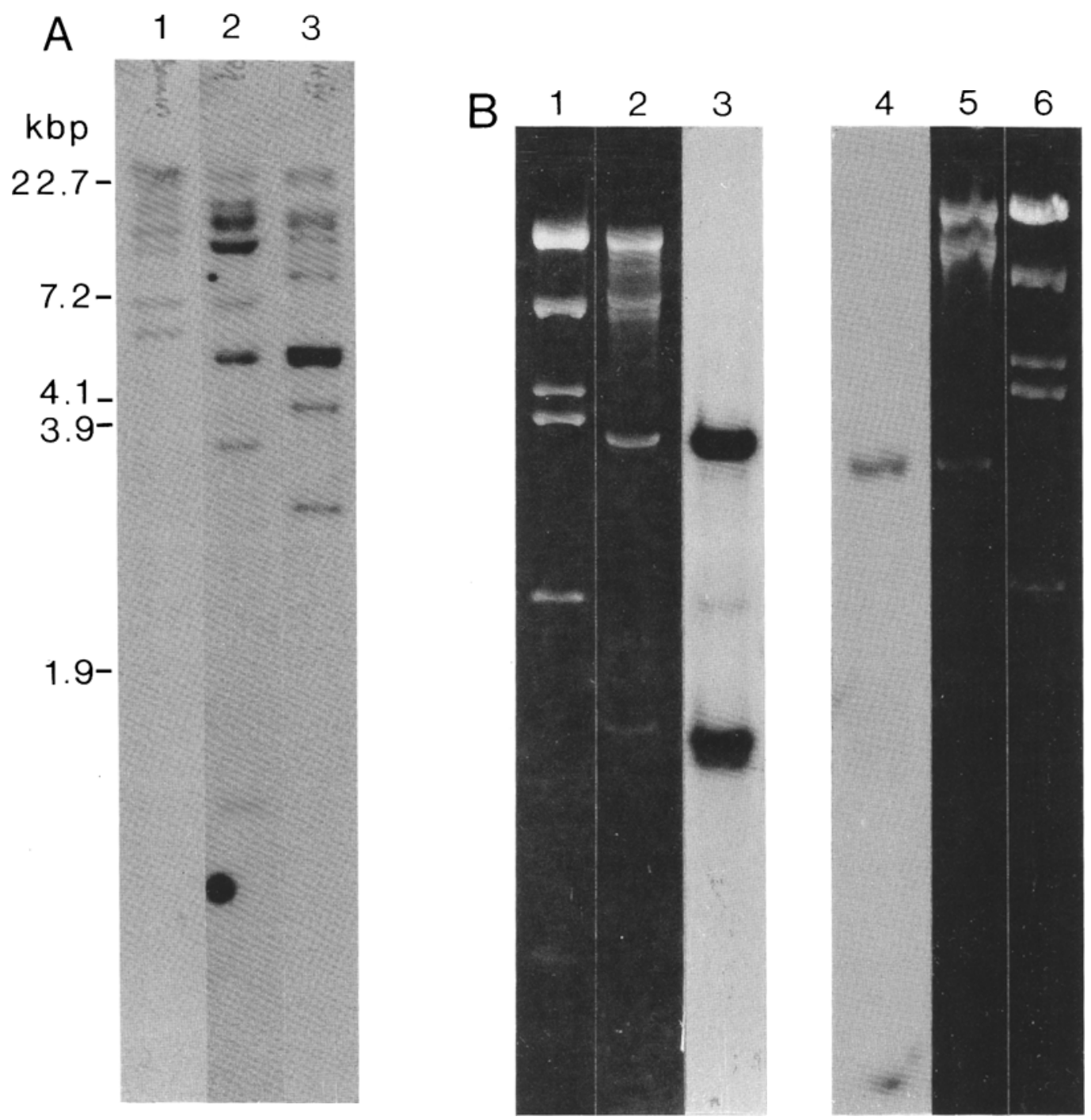

Figure 1. Southern blot analysis of Carlsberg II DNA (A) and clone $\lambda$ hor $2-4$ (B).

Restriction fragments of endosperm DNA (A) and $\lambda$ hor2-4 DNA (B) were separated by agarose gel electrophoresis, transferred to nitrocellulose filters and hybridized to ${ }^{32} \mathrm{P}$-labelled pc hor $2-4$ DNA. The hybridizing fragments were detected by autoradiography. The sizes of the fragments were estimated using the 22.7, 7.2, 4. 1, 3.9 and $1.9 \mathrm{kbp}$ Hind III restriction fragments of $\lambda g t$ wes. $\lambda B$ as markers.

1A. Carlsberg II DNA restriction fragments. Autoradiogram. Track 1: Bam HI; track 2: Eco RI; track 3: Hind III. 1B. $\lambda$ hor 2-4 DNA restriction fragments. Tracks 1, 6: size markers; tracks 2, 3: Eco RI; tracks 4, 5: Hind III; tracks $1,2,5,6$ : ethidium bromide stained agarose gel; tracks 3,4 : autoradiogram.

the gene, $\lambda$ hor $2-4$, and that the latter is active in the developing endosperm. There is no resemblance to the first 17 amino acids predicted by the pc hor $2-4$ cDNA and we consider this portion of the clone to be the result of unfaithful reverse transcription.
Hordein polypeptides are synthesized on membrane-bound polysomes of the endoplasmic reticulum. These primary translation products are co-translationally transported and cleaved to yield mature hordein polypeptides which are $2 \mathrm{kD}$ smaller than their precursors (3, 


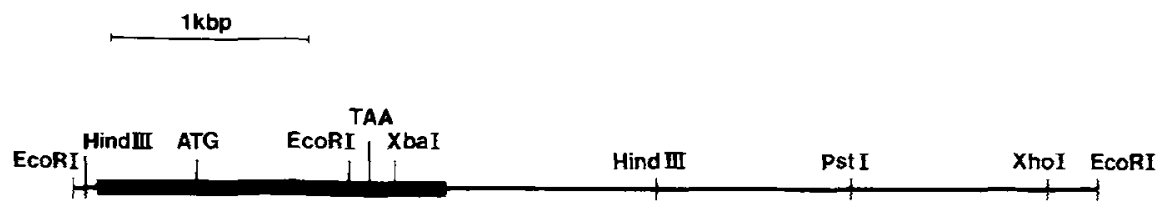

Figure 2. Restriction map of the $5 \mathrm{kbp}$ Eco RI fragment containing the Bl hordein gene. The heavy line indicates the sequenced region. A.TG = start methionine, TAA = stop codon.

6, 40). The first 19 amino acids encoded by the open reading frame are considered to be the signal peptide, as the sequence is consistent with the common features of known signal peptides (16). A charged lysine residue lies adjacent to the initiator methionine and a core of hydrophobic amino acids is present. Since purified Bl hordein polypeptides are inaccessible to Edman degradation (34) amino terminal sequences are not available. The most likely signal peptide cleavage point lies between alanine 19 (5 residues after the core) and glutamine 20. The following 159 nucleotides encode 9 blocks of 4 to 6 proline and glutamine residues (Figure 4 ). There are in all 21 glutamine and 21 proline residues in these blocks, which are separated by one or two other amino acid residues. Six of the blocks are linked to their neighbour with phenylalanine, two with tyrosine and one with isoleucine. In the nine blocks the core sequence PQQP is preferred to other arrangements. This domain of 53 amino acids has thus a content of
$78 \%$ glutamine and proline residues. In the amino acid sequence deduced from the following stretch of 492 nucleotides the frequency of glutamine and proline residues drops to $41 \%$ but they still tend to be organized in blocks separated by longer peptides containing other residues. In this domain 52 glutamines are associated with 18 prolines. It is to be noted that 7 of the 8 cysteine residues encoded by the gene are in this latter region and leucine and serine are frequent neighbours to glutamine residues. The 35 carboxy-terminal amino acids encoded by the gene lack glutamine residues and contain only 3 proline residues. Codon usage in the $\lambda$ hor $2-4$ gene is remarkable by the preferential use of the CAA codon for glutamine (74\%) and the CCA codon for proline (77\%) (Figure 5). Otherwise the codon usage is not biased.

The overall G-C content of the sequence is $44 \%$. The coding region has a $\mathrm{G}-\mathrm{C}$ content of $48 \%$ whereas the 5 ' and 3 ' non-coding regions have $37 \%$ and $41 \%$, respectively. In the 5 ' non-

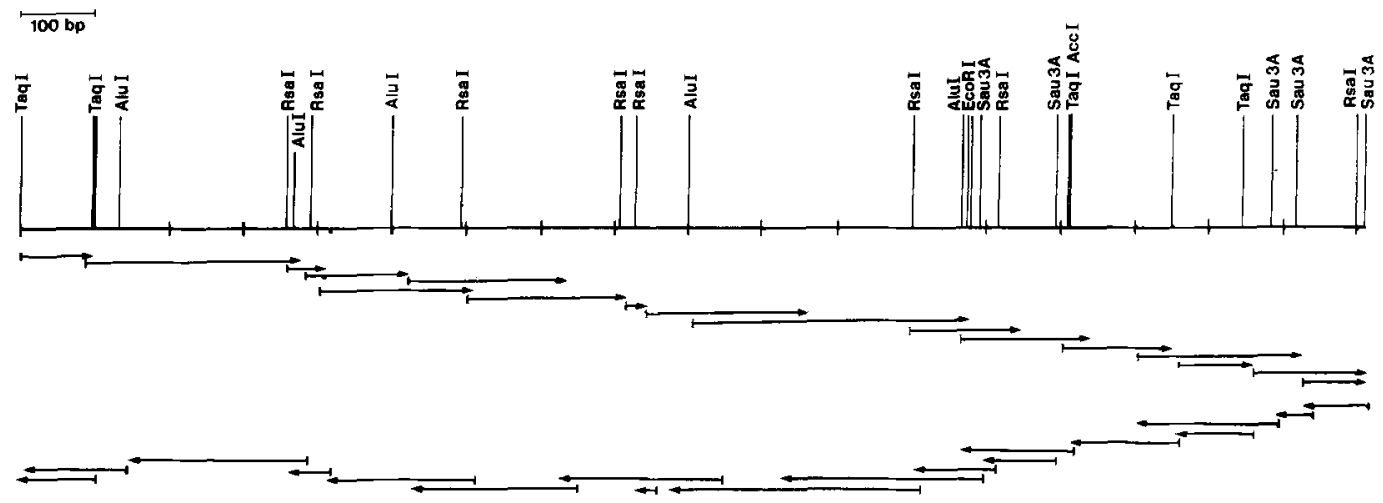

Figure 3. Restriction fine map and strategy used to determine the 1775 bp sequence. 
A. BRANDT et al.: B1 hordein gene

$-550$

$-500$

GTGCAGgTGT ATGAGTCATT GTTATGATCT ATAGgTGTCA GTTTATCTTA TCATCTGGGT GATCAATACA $-400$

GGCCCAGGTT TTATAAAAAC CAGTCGAGTC GAGAAGAACC GTCCACATGT AAAGCTTTAA CAACCCACAC ATTGATTGCA

ACTTAGTCCT ACACAAGTTT TCCATTCTTG ITTCAGGCTA ACAACCTATA CAAGGTTCCA AAATCATGCA AAAGTGATGC

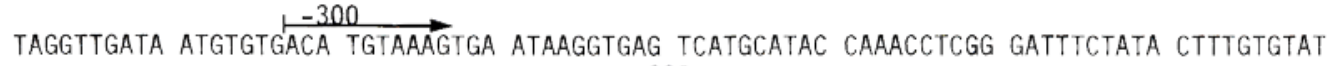
$-200$

GATCATATGC ACAACTAAAA GGCAACTTTG ATTATCAATT GAAAAGTACC GCTTGTAGCT TGTGCAACCT AACACAATGT $-100$

CCAAAAATCC ATTTGCAAAA GCATCCAAAC ACAATTGTTA AAGCTGTTCA AACAAACAAA GAAGAGATGA AGCCTGGCTA unn CTATAAATAG GCAGGTAGTA TAGAGATCTA CACAAGCACA AGCATCAAAA CCAAGAAACA CTAGTTAACA CCAATCCACT unnus

$+1$

ATG AAG ACC TTC CTC ATC TTT GCA CTC CTC GCC ATT GCG GCA ACA AGT ACG ATT GCA CAG CAA CAA met lys thr phe leu ile phe ala leu leu ala ile ala ala thr ser thr ile ala gln gln gln 100

CCA TTT CCA CAA CAA CCC ATC CCA CAA CAG CCA CAA CCA TAC CCA CAA CAA CCA CAA CCA TAT CCA pro phe pro gln gln pro ile pro gln gln pro gln pro tyr pro gln gln pro gln pro tyr pro

CAA CAA CCC TTC CCA CCG CAA CAA GCT TTC CCA CAA CAA CCA CCA TTT TGG CCA CAA CAA CCA TTT gln gln pro phe pro pro gln gln ala phe pro gln gin pro pro phe trp pro gln gin pro phe 200

CCA CAG CAA CCA CCA TTT GGG CTA CAA CAA CCA ATT CTG TCG CAA CAA CAA CCA TGT ACA CCA CAA pro gln gln pro pro phe gly leu gln gln pro ile leu ser gln gln gln pro cys thr pro gln 300

CCA ACA CCA CTC CCA CAA GGA CAA CTG TAC CAA ACG CTT CTG CAA CTA CAA ATA CCC TAT GTT CAA gln thr pro leu pro gln gly gln leu tyr gln thr leu leu gln leu gln ile pro tyr val gln

CCA TCT ATT TTG CAA CAG CTA ACC CCA TGC AAG GTA TTC CTC CAG CAG CAG TGC AGC CCC GTG CGA pro ser ile leu gln gln leu asn pro cys lys val phe leu gln gln gln cys ser pro val arg 400

ATG CCA CAA CTT ATT GCT AGG TCG CAA ATG TTG CAG CAG AGC AGT TGC CAT GTG TTG CAG CAA CAA met pro gln leu ile ala arg ser gln met leu gln gln ser ser cys his val leu gln gln gln TGT TGC CAG CAA CTG CCG CAA ATC CCC GAA CAA TTC CGC CAT GAG GCA ATC CGT GCA ATC GTC TAC cys cys gln gln leu pro gln ile pro glu gln phe arg his glu ala ile arg ala ile val tyr

TCT ATC TTT CTG CAA GAA CAA CCC CAA CAG TCG GTC CAA GGT GCC TCC CAA CCC CAA CAA CAG TTG ser ile phe leu gln glu gln pro gln gln ser val gln gly ala ser gln pro gln gln gln leu 600

CAG GAG GAG CAA GTC GGA CAA TGT TAT TTC CAA CAA CCT CAA CCA CAA CAA CTT GGT CAA CCA CAA gln glu glu gln val gly gln cys tyr phe gln gln pro gln pro gln gln leu gly gln pro gln CAG GTA CCA CAG AGT GTT TTC TTG CAG CCA CAC CAG ATA GCT CAG CTT/GAG GCC ACG AAT TCC ATT gln val pro gln ser val phe leu gln pro his gln ile ala gln leu|glu ala thr asn ser ile

GCG CTG CGT ACC CTA CCA ACG ATG TGC AAT GTT AAT GTG CCA TTG TAT GAC ATC ATG CCA TTC GGC ala leu arg thr leu pro thr met cys asn val asn val pro leu tyr asp ile met pro phe gly $800 \quad 813$

GTT GGC ACT AGA GTT GGT GTC TAA TGATAAGAAA AGgTCTCTAG AAATATATAG TTGgATCCGg TTGTTCTAGT val gly thr arg val gly val

900

CGATGTAGCG GTGACAAATA AAGTGTCACA CAACCTTATG TGTGACCAGC CGAAACTAGT TGTTTAAATT TTGAAATAAA TATAAATAAA GTTCATGACG ACTATCTGAA AAGTTTCTCG AACAAGTCGA AAACTGTATT AATTCCTCCC CGACCCTGCC AAACCGAATG ACTAACTGAA AGACAGATGT ATCTACATCG ACCCCTTTGC TCAAAATGAC ATTCTTTTGG TGGACAGCGG AGATTCAGAA TCCTGCCGTC AAGTTTTGTC AGATCCATCC AAGGATGICG TTCCCATTGT TCGTCATGGC CTCTAACAAT $1200 \quad 1225$

AAACAGCCTC TTGAGGACCC CTCTCGACCT GAACGGTAC 
Figure 4. The sequence containing the $B I$ hordein gene. The first nucleotide in the open reading frame is numbered +1 . Horizontal arrows above the nucleotides indicate repeats and inverted repeats in the sequence. The vertical arrow between 57 and 58 indicates the most likely signal peptide cleavage point. The vertical lines at position 216 and 708 divide the polypeptide into 3 regions with distinct composition. Wavy lines below the nucleotides in the flanking regions mark possible regulatory sequences.

\section{Second letter}

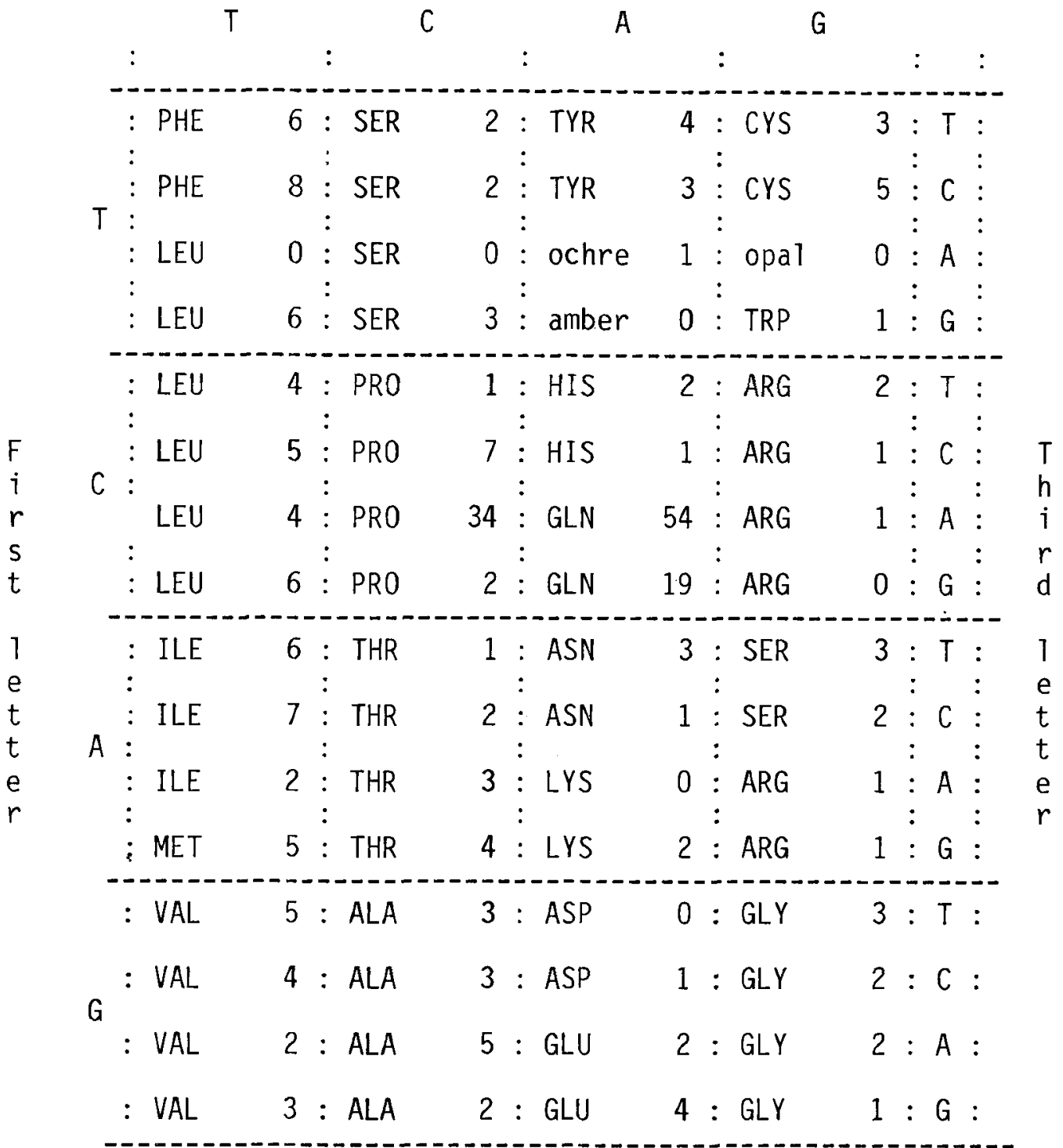

Figure 5. The codons used by the $\lambda$ hor $2-4$ gene. 
coding region at position -79 the TATA-box like sequence, TATAAATA, is present. Sequences ACAATT, ATCCAA, TGCAAA and GTCCAAA are found in the region from -130 to -160 and may represent CAAT-boxes. Eight different repeated sequences of 8 to 10 nucleotides in length are found in the 5 ' non-coding region (Figure 4). The sequence CAAA is directly repeated 3 times at position -110 and the sequence CACAAG is directly repeated around position -50. The decanucleotide ACATGTAAAG is repeated 2 times at positions -436 and -297 .

The 3' non-coding region contains three putative polyadenylation signals, AATAAA. Eleven nucleotides downstream from the third polyadenylation signal, at the position where most cDNAs contain a poly A tail, lies a nearly perfect 14 nucleotide inverted repeat. The sequence allows a secondary structure to be formed which may be important for the termination of transcription and/or polyadenylation of the transcript (Figure 6).

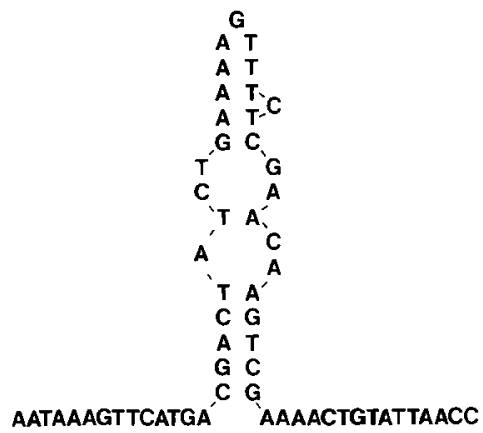

Figure 6. A possible loop structure which can be formed next to the polyadenylation signal. The arrows indicate the position where the poly-A tail is located in most cDNA clones.

\subsection{Nucleotide and amino acid sequence heterogeneity within the $B$ hordein gene family}

The nucleotide sequence of the gene $\lambda$ hor $2-4$, in the 3' half of the coding region can be compared with that of three different partial cDNA clones encoding B1 hordein polypep- tides, pc hor2-5, 2-7 and 2-8 (Figure 7). All 4 sequences are.homologous but differ by a num. ber of nucleotide substitutions. In addition, all 3 cDNA clones have one triplet less than $\lambda$ hor2-4. In the 3 ' non-coding region a deletion of 27 nucleotides is apparent in clone pc hor $2-8$ relative to $\lambda$ hor2-4, pc hor2-5 and 2-7, whereas clone $\lambda$ hor $2-4$ suffers a smaller deletion of 11 nucleotides in comparison with the three cDNA clones. In relation to $\lambda$ hor $2-4$ the known parts of the coding regions for clones pc hor 2-5, 2-7 and 2-8 have 39,13 and 40 nucleotide substitutions, respectively, which results in 21,13 and 14 amino acid substitutions. The four sequences thus encode 4 different $\mathrm{B} 1$ hordein polypeptides.

\section{DISCUSSION}

The B hordein polypeptides from the endosperm of Carlsberg II barley have molecular weights from $30-38 \mathrm{kD}$, the most prominent polypeptides being $\mathrm{B} 1$ with $-30 \mathrm{kD}$ and $\mathrm{B} 3$ with $\sim 38 \mathrm{kD}$. Peptide sequence information is only available for the $\mathrm{B} 1$ polypeptides (34) and since the $29 \mathrm{kD}$ polypeptide encoded by the gene $\lambda$ hor 2-4 matches closely with the peptide information available we infer that the hor $2-4$ gene encodes a Bl hordein polypeptide. Peptide mapping of purified B hordein polypeptides ( 9 , 18 ) suggests that the $B 1$ and the $B 3$ polypeptides are closely related, but of different structure. We have failed to discriminate, even at stringent hybridization conditions $\left(\mathrm{Tm}-8^{\circ} \mathrm{C}\right)$ between the two size classes of mRNAs (1200 and 1400 bases) (19) which should encode B1 and B3 polypeptides, respectively. On the other hand two cDNA clones $\mathrm{pB} 7$ and $\mathrm{pB} 11$ have been shown by hybrid release translation experiments to encode $\mathrm{B} 3$ and $\mathrm{B} 1$ hordein polypeptides, respectively (11). The clone pc hor2-8 (Figure 7) aligns closely with the clone $\mathrm{pB} 11$ and the clones pc hor2-5 and pc hor2-7 (Figure 7) with the clone pB7. Our failure to discriminate among B1 and B3 mRNAs by hybridization thus reflects the high degree of homology between the different $B$ hordein sequences.

Recently the sequence of another B1 hordein genomic clone, $\lambda \mathrm{HvBH} 3.4$, has been published (12). $\lambda \mathrm{HvBH} 3.4$ was identified as a BI hordein gene firstly by virtue of its coding capacity and 
secondly by its close sequence homology to clone $\mathrm{pB} 11$, a B1 hordein cDNA clone (11). $\lambda$ hor 2-4, which also has a coding capacity for a B1 hordein, shows a high sequence homology to $\lambda$ $\mathrm{HvBH} 3.4$ over the 1775 bp that can be compared. The homology is greatest in the 5 ' flanking region with single nucleotide substitutions being less than $10 \%$. In the coding region and 3 ' flanking region, $\mathrm{pHvBH} 3.4$ aligns with cDNA pc hor2-8 (which is similar to pB11) and contains the same deletions with respect to the $\lambda$ hor 2-4 in the 3 ' flanking region (Figure 7). It appears that although $\lambda$ hor $2-4$ is closely homologous with $\mathrm{pB} 7$ it could not encode a polypeptide as large as a B3 hordein. A detailed comparison of the coding regions of the $2 \mathrm{~B}$ hordein genomic clones reveals the following differences. First, the gene $\lambda$ hor $2-4$ has a $69 \mathrm{bp}$ deletion in the amino terminal domain 1 , with the blocks of proline-glutamine residues, compared to $\mathrm{HvBH} 3-4$ resulting in a 23 amino acid shorter B1 hordein polypeptide. This may indicate that insertions and deletions of such blocks in the amino terminal region are the major source of the size polymorphisms within the B hordein polypeptide family. Fifty-four additional nucleotide changes in the remaining coding region result in 24 amino acid substitutions. Seven of these substitutions change a neutral amino acid to a charged residue. Thus, point mutations in the second domain with the scattered glutamine-proline blocks may be the major reason for the charge heterogeneity (9) observed among $B$ hordein polypeptides. The two genes are members of the hordein gene cluster and encode two different B 1 hordein polypeptides. The remarkable sequence conservation observed in the 5' upstream and 3' downstream regions between the two $B$ hordein genes is potentially of regulatory importance. Comparable extensive 5' upstream sequence homology has also been observed between 3 genes for pea legumin, and may reflect conservation of regulatory sequences for the coordinate expression of members of the gene families (25).

Different variations of glutamine-proline blocks (Figure 4) are found in the amino-terminal part of the $\alpha / \beta$ gliadin storage proteins of wheat $(1,29,38)$. The clustering of glutamineproline blocks in the amino-terminal region is not a feature of maize storage protein, zein (20, $28)$ or the high MW glutenin of wheat $(13,39)$.

Un-biased codon usage in the $\lambda$ hor 2-4 gene, (exceptions CAA and CCA) is in sharp contrast to the codons used by the $\alpha$-amylase (31) and $(1 \rightarrow 3,1 \rightarrow 4)$ - $\beta$-glucanase $(10)$ genes which are expressed in the aleurone of the germinating barley grain. These genes preferentially use $C$ or $\mathrm{G}$ in the wobble position resulting in a remarkably high GC content in the coding region. The aleurone has the same developmental origin as the endosperm and is also active during grain filling (7). The tRNA charging capacity in the grain may help to prevent expression of these two germination specific genes during grain development.

Examination of the 5' non-coding region reveals a typical TATA box, TATAAATA, which may act as a promoter, and several small repeats. Among these is the sequence, ACATGTAAAG, which is repeated twice. Part of this sequence has been found by LYCETT et al. (24, 25 ) in the 5' upstream region of the Leg A, B and $C$ genes in pea. The sequence is also present in the wheat $\boldsymbol{\alpha} / \boldsymbol{\beta}$ gliadin genes $(29,38)$, the maize zein genes $(20,28)$ and a variant (TCTAAAG) in the wheat high $\mathrm{MW}$ glutenin gene $(13,39)$. As noted (24) this sequence is highly homologous to the core sequence of the SV 40 enhancer (41).

The lack of a transformation and expression system in barley prevents the use of deletion analysis and site-directed mutagenesis in $\lambda$ hor 2 4 , to study the importance of specific regulatory sequences in the 5' and 3' flanking regions. Transgenic expression has been achieved with the gene encoding the small subunit of ribulose bisphosphate carboxylase from pea in Petunia (4) and with the phaseolin gene from Phaseolus vulgaris in tobacco (35). In both cases the foreign genes are expressed in the correct tissue under the developmental control of their own 5 ' flanking region. Similar attempts to express the $\lambda$ hor 2-4 gene in tobacco may be initiated with the aim of characterizing regulatory sequences in the 5' and 3' untranslated region of the hordein gene. Systems are available for the in vitro transcription of cloned genes. It is tempting to use site-directed mutagenesis to introduce specific changes in the coding region of the hor $2-4$ gene. Reconstitution of these transcripts into 
A. BRANDT et al:: $\mathrm{Bl}$ hordein gene
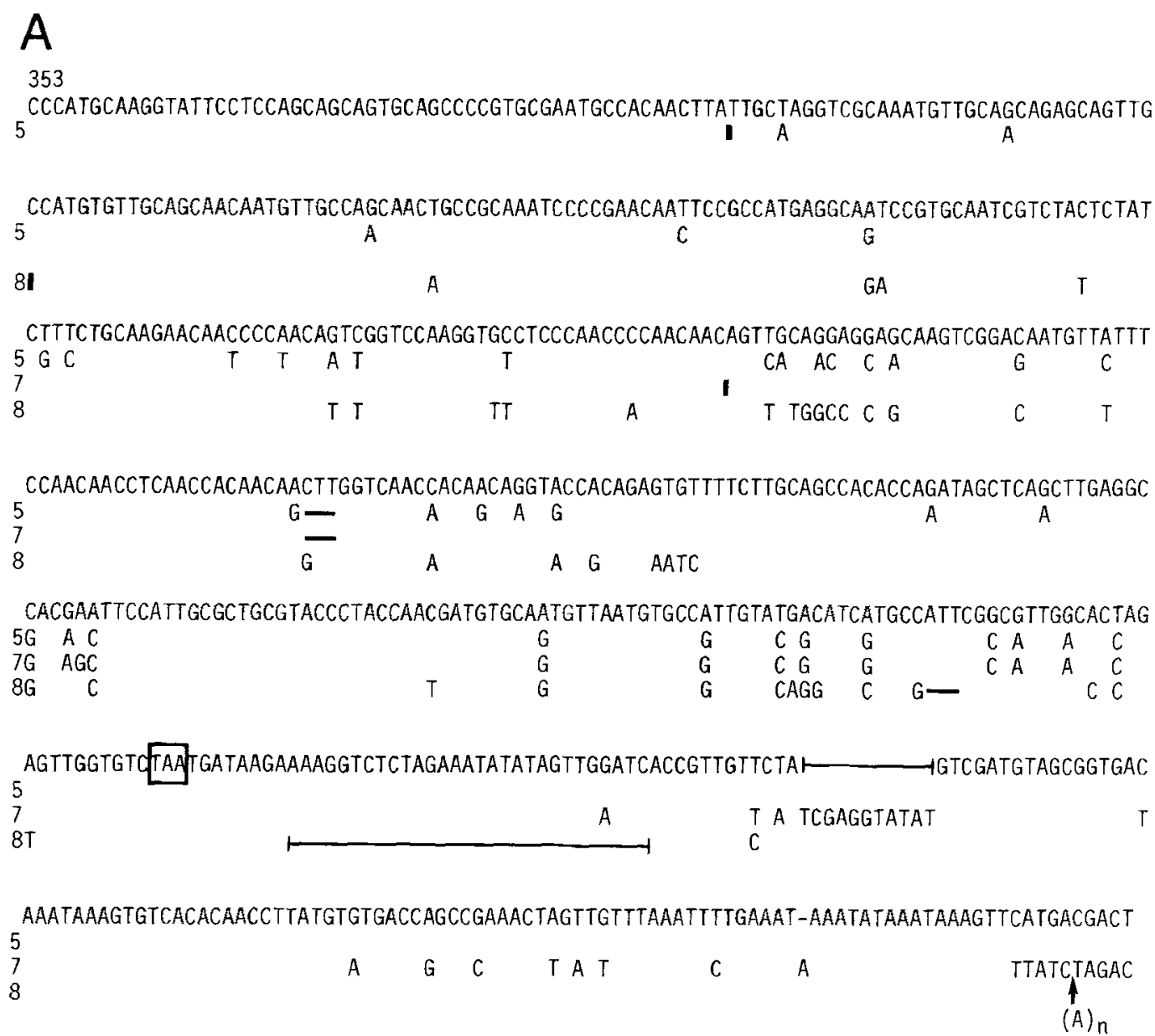

Figure 7. Nucleotide (A) and deduced amino acid (B) sequence heterogeneity of B1 hordein polypeptides. The clone $\lambda$ hor 2-4 which has sequence identity to the cDNA clone pc hor 2-4 is compared from position 353 with 3 different B1 hordein cDNAs. 5: pc hor 2-5, 7: pc hor 2-7, 8: pc hor 2-8. Vertical bars indicate the start of the cDNA sequences, horizontal lines signify deletions. The stop codon is boxed.

rough microsomes $(6,40)$ may then be used to study regions of the $\mathrm{B} 1$ hordein polypeptide important for signal peptide cleavage and cotranslational transport.

\section{ACKNOWLEDGEMENTS}

We thank J. ENTWISTLE and T, BEKTVED for excellent technical assistance.

The work was supported by the Biomolecular Engineering Programme of the Commission of the European Communities with training con- tract GBI-123-DK to V. CAMERON-MILLS and contract no. GBI 1-4-024-Dk to Professor D. VON WETTSTEIN.

\section{REFERENCES}

1. Anderson, O. D. J C. Litts, M.-F. Gautier \& F. C. GREENE: Nucleic acid sequence and chromosome assignment of a wheat storage protein gene. Nucl. Acids Res. 12, $8129-8144$ (1984)

2. Benton. W. D. \& R. W. Davis: Screening $\lambda \mathrm{gt}$ recombinant clones by hybridization to single plaques in situ. Science 196, 180-182 (1977) 
A. BRANDT et al.: B1 hordein gene

B

ile ala arg ser gln met leu gln gln ser ser cys his val leu gln gln 5

gln cys cys gln gln leu pro gln ile pro glu gln phe arg his glu ala 5 8

ile arg ala ile val tyr ser ile phe leu gln glu gln pro gln gln ser 5 val val 8 his

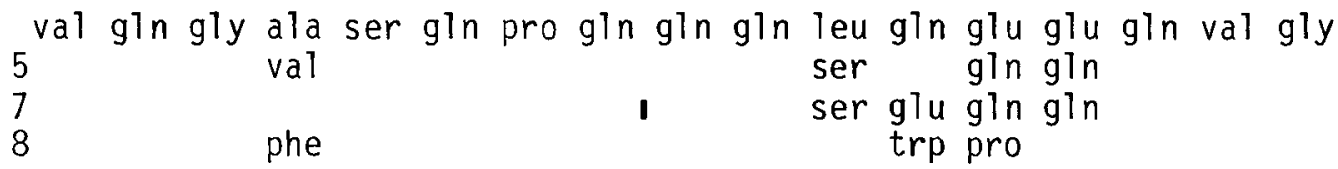

gln cys tyr phe gln gln pro gln pro gln gln leu gly gln pro gln gln $5 \mathrm{glu}$ ser 7 ser 8 phe $\longmapsto \quad g l n$ val

val pro gln ser val phe leu gln pro his gln ile ala gln leu glu ala 5

7

8 Tys ser

thr asn ser ile ala leu arg thr leu pro thr met cys asn val asn val 5 thr 7 ala ser

8 thr met

pro leu tyr asp ile met pro phe gly val gly thr arg val gly val

5 arg val leu ala ile asp

7 val leu ala ile asp

8

leu arg pro ser

3. BRANDT A. \& J. INGVERSEN: Isolation and translation of hordein messenger RNA from wild type and mutant endosperm in barley. Carlsberg Res. Commun. 43, $451-469$ (1978)

4. Broglie, R. G. Coruzzi, R. T. Fraley, S G. Rogers, R. B. HorsCh, J. G. Niedermeyer, C. L. FINK. J. S. FLICK \& N.-H. CHUA: Light-regulated expression of a pea ribulose-1,5-bisphosphate carboxylase, small subunit gene in transformed plant cells. Science 224, 838-843 (1984)

5. CAMERON-Mills, V.: The structure and composition of protein bodies purified from barley endosperms by silica sol density gradients. Carlsberg Res. Commun. 45, 557-576 (1980)
6. Cameron-Mills, V. \& J. IngVersen: In vitro synthesis and transport of barley endosperm proteins: Reconstitution of functional rough microsomes from polyribosomes and stripped microsomes. Carlsberg Res. Commun. 43, $471-489$ (1978)

7. Cameron-Mills, V. \& D. von Wettstein: Protein body formation in the developing barley endosperm. Carlsberg Res. Commun. 45, 577-594 (1980)

8. Covarrubias. L., L. Cervantes, A. CovarRubias, X. Soberón, I. Vichido. A. Blanco, Y, M. Kupersztoch-Portnoy \& F. Bolivar: Construction and characterization of new cloning ve- 
hicles, V. Mobilization and coding properties of pBR322 and several deletion derivatives including pBR327 and pBR328. Gene 13, 25-35 (1981)

9. Faulks. A. J.. P. R. Shewry \& B J Miflin: The polymorphism and structural homology of storage polypeptides (hordein) coded by the Hor 2 locus in barley (Hordeum vulgare L. ). Biochem. Genet. 19, 841-858 (1981)

10. Fincher, G.-B., P. A. Lock. M. M. Morgan, K. Lingelbach. R. E. H. Wettenhall. J. F. B. Mer CER. A BRANDT \& K. K. ThOMSEN: Primary structure of the $(1 \rightarrow 3,1 \rightarrow 4)$ - $\beta$-glucanase from barley aleurone. Proc. Natl. Acad. Sci. USA (in press)

II. Forde. B. G.. M. Kreis. M. S. Williamson. R. P. Fry, J Pywell, P. R. Shewry, N. Brunce \& B. J. MifLIN: Short tandem repeats shared by B- and C-hordein cDNAs suggest a common evolutionary origin for two groups of cereal storage protein genes. The EMBO J. 4, 9-15 (1985)

12. Forde. B. G.. A. Heyworth J. Pywell \& M. KREIS: Nucleotide sequence of a B1 hordein gene and the identification of possible upstream regulatory elements in endosperm storage protein genes from barley, wheat and maize. Nucl. Acids Res. 13, 7327-7339 (1985)

13. Forde. J.. J.-M. Malpica. N. G. Halford. P. R. Shewry, O. D. Anderson, F. C. Greene \& B. J MIfLIN: The nucleotide sequence of a HMW glutenin subunit gene located on chromosome 1A of wheat (Triticum aestivum L. ). Nucl. Acids Res. 13, 6817-6832 (1985)

14. Giese. H.. B. Andersen \& H. Doll: Synthesis of the major storage protein, hordein, in barley. Pulse-labeling study of grain filling in liquid-cultured detached spikes. Planta 159, 60-65 (1983)

15. GiESE. H. \& H. E. HoPP: Influence of nitrogen nutrition on the amount of hordein, protein $Z$ and $\beta$-amylase messenger RNA in developing endosperms of barley. Carlsberg Res. Commun. 49, 365-383 (1984)

16. HEIJNE, G. voN: On the hydrophobic nature of signal sequences. Eur. J. Biochem. 116, 419-422 (1981)

17. HoHn, B. \&. MURRAY: Packaging recombinant DNA molecules into bacteriophage particles in vitro. Proc. Nat. Acad. Sci. 74, 3259-3263 (1977)

18. Holder. A. A. \& J. Ingversen: Peptide mapping of the major components of in vitro synthesized barley hordein: Evidence for structural homology. Carlsberg Res. Commun. 43, $177-184$ (1978)

19. Hopp. H. E.. S. K. Rasmussen \& A. BrandT: Organization and transcription of $B 1$ hordein genes in high lysine mutants of barley. Carlsberg Res. Commun. 48, 201-216 (1983)

20. Hu. N.T. M. A. Peifer, G. Heidecker, I. Mes-
SING \& I. RUBENSTEIN: Primary structure of a genomic zein sequence of maize. The EMBO J. 1, 1337-1342 (1982)

21. Jensen. I. H. J. Jørgensen. H. P. Jensen, H. Giese \& H. DOLL: Linkage of the hordein Ioci Horl and Hor2 with the powdery mildew resistance loci Ml-k and Ml-a on barley chromosome 5. Theor. Appl. Genet. 58, 27-31 (1980)

22. Kreis, M. P. R. Shewry. B. G. Forde. S. RaHMAN \& B. J. MIflin: Molecular analysis of a mutation conferring the high-lysine phenotype on the grain of barley (Hordeum vulgare). Cell 34, 161-167 (1983)

23. Loenen. W. A. M. \& W. J. Brammer: A bacteriophage lambda vector for cloning large DNA fragments made with several restriction enzymes. Gene 10, 249-259 (1980)

24. Lycett, G. W. R. R. D. Croy, A. H. Shirsat \& D. BOUlter: The complete nucleotide sequence of a legumin gene from pea (Pisum sativum L.). Nucl. Acids Res. 12, 4493-4506 (1984)

25. Lycett. G. W. R. R. D. Croy, A. H. Shirsat, D. M. RICHARDS \& D. BOUlter: The $5^{\prime}$-flanking regions of three pea legumin genes: comparison of the DNA sequences. Nucl. Acids Res. 13 , 6733-6743 (1985)

26. Maniatis. T.. E. F. Fritsch \& J. Sambrook: Molecular cloning, a laboratory manual. Cold Spring Harbor Laboratory (1982)

27. MEssing.J.\& J. VIEIRA: A new pair of M 13 vectors for selecting either DNA strand of double-digest restriction fragments. Gene 19, 269-276 (1982)

28. Pedersen. K. J. Devereux. D. R. Wilson, E. SHELDON \& B. A. LARKINS: Cloning and sequence analysis reveal structural variation among related zein genes in maize. Cell 29, $1015-1026$ (1982)

29. Rafalski. J. A. K. Scheets, M. Metzler. D. M. Peterson. C. Hedgcoth \& D Soll: Developmentally regulated plant genes: The nucleotide sequence of a wheat gliadin genomic clone. The EMBO J. 3, $1409-1415$ (1984)

30. Rasmussen. S. K.. H. E. Hopp \& A. Brandt: Nucleotide sequences of cDNA clones for $B 1$ hordein polypeptides. Carlsberg Res. Commun. 48, 187 199 (1983)

31. Rogers, J. C, \& C. Milliman: Isolation and sequence analysis of a barley $\alpha$-amylase cDNA clone. J. Biol. Chem, 258, 8169-8174 (1983)

32. Sanger. F. S. Nicklen \& A. R. Coulson: DNA sequencing with chain-terminating inhibitors. Proc. Nat. Acad. Sci. USA 74, 5463-5467 (1977)

33. Sснмітт, J. M.: Purification of hordein polypeptides by column chromatography using volatile solvents. Carlsberg Res. Commun. 44, 431-438 (1979) 
A. BRANDT et al.: B1 hordein gene

34. Schmitt. J. M. \& I. Svendsen: Partial amino acid sequence from hordein polypeptide B1. Carlsberg Res. Commun. 45, 549-555 (1980)

35. Sengupta-Gopalan, C. N. A Reichert. R. F. Barker. T. C. Hall \& J. D. Hemp: Developmentally regulated expression of the bean $\beta$ phaseolin gene in tobacco seed. Proc. Natl. Acad. Sci. USA 82, 3320-3324 (1985)

36. Shewry. P. R., A. J. Faulks. R. A. Pickering, I. T. JONES. R. A. Finch \& B. J. Miflin: The genetic analysis of barley storage proteins. Heredity 44 , 383-389 (1980)

37. Souciet. J.-L., J.C. Hubert \& F. LaCroute: Cloning and restriction mapping of the yeast URA2 gene coding for the carbamyl phosphate synthetase aspartate-transcarbamylase complex. Mol. Gen. Genet. 186, 385-390 (1985)
38. Sumner-Smith, M. J. A. Rafalski. T Sugiyama, M. Stoll \& D. Söll: Conservation and variability of wheat $\alpha / \beta$-gliadin genes. Nucl. Acids Res. 13, 3905-3916 (1985)

39. Thompson. R. D.. D. Bartels \& N. P. HARBerd: Nucleotide sequence of a gene from chromosome 1D of wheat encoding a HMW-glutenin subunit. Nucl. Acids Res. 13, 6833-6846 (1985)

40. Weber. E. \& A. Brandt: Species specific signal peptide cleavage of plant storage protein precursors in the endoplasmic reticulum. Carlsberg Res. Commun. 50, 229-308 (1985)

41. WeIher. H. M. KöNIG \& P. Gruss: Multiple point mutations affecting the simian virus 40 enhancer. Science 219, 626-631 (1983)

Accepted by E. LUND 